bioRxiv preprint doi: https://doi.org/10.1101/2020.09.14.297325; this version posted September 15,2020 . The copyright holder for this preprint

(which was not certified by peer review) is the author/funder. All rights reserved. No reuse allowed without permission.

\title{
Assessing and Predicting Efficacy of Dance Intervention for Parkinson's Disease
}

Anna Krotinger, Wesleyan University and The Harvard Medical School Center for Bioethics, and Psyche Loui, Northeastern University and Wesleyan University 


\begin{abstract}
Parkinson's disease (PD) is associated with a loss of internal cueing systems, affecting rhythmic motor tasks such as walking and speech production. Music and dance encourage spontaneous rhythmic coupling between sensory and motor systems; this has inspired the development of dance programs for PD. Here we assessed the therapeutic outcome and some underlying cognitive mechanisms of dance classes for PD, as measured by neuropsychological assessments of disease severity as well as quantitative assessments of rhythmic ability and sensorimotor experience. We assessed prior music and dance experience, beat perception (Beat Alignment Test), sensorimotor coupling (tapping to high- and low-groove songs), and disease severity (Unified Parkinson's Disease Rating Scale in PD individuals) before and after four months of weekly dance classes. PD individuals performed better on UPDRS after four months of weekly dance classes, suggesting efficacy of dance intervention. Greater post-intervention improvements in UPDRS were associated with the presence of prior dance experience and with more accurate sensorimotor coupling. Prior dance experience was additionally associated with enhanced sensorimotor coupling during tapping to both high-groove and low-groove songs. These results show that dance classes for PD improve both qualitative and quantitative assessments of disease symptoms. The association between these improvements and dance experience suggests that rhythmic motor training, a mechanism underlying dance training, impacts improvements in parkinsonian symptoms following a dance intervention.
\end{abstract}

\title{
Keywords
}

Parkinson's disease, dance, music, intervention, longitudinal, rhythm 


\section{Introduction}

Parkinson's disease (PD) is a neurodegenerative disorder characterized by motor symptoms including tremor, rigidity, and akinesia, which affect daily activities such as walking and speaking (Lang \& Lozano, 1998). The cognitive mechanisms underlying the motor symptoms likely involve a loss of internal cueing systems, which are evident from motor tasks such as walking, finger tapping, and musical rhythm perception (Hausdorff et al., 1998; Grahn, 2009; Mirelman et al., 2019; Rose et al., 2020). These tasks all depend on rhythmic timing, either emergent or event-based, through rhythmic movement or entrainment to an external cue (Spencer \& Ivry, 2005). The perception of rhythmic timing and subsequent production of rhythmic movement are dependent on dopaminergic activity in the corticostriatal circuits (Meck, 1996; Meck \& Benson, 2001; Nenadic et al., 2003; Jahanshahi, et al. 2006), including dopaminergic cell loss in the substantia nigra pars compacta, resulting in diminished levels of available dopamine (Poewe et al., 2017). As a result, PD patients exhibit decreased activation of the basal ganglia when listening to music, as compared with healthy adults (Grahn \& Brett, 2009), in addition to deficits in both rhythmic perception and production (Pastor et al., 1992; O'Boyle et al. , 1996; Hellström et al., 1997; Spencer \& Ivry, 2005, Grahn \& Brett, 2009).

Music- and dance-based interventions, which provide external auditory and visual cues, have been explored as a means of supplementing the timing and cueing deficits resulting from basal ganglia impairment in PD (Sharp \& Hewitt, 2014; Benoit et al., 2014; Dalla Bella et al., 2015; Devlin et al., 2019). One specific type of intervention is Rhythmic Auditory Stimulation (RAS), in which participants walk to an external auditory stimulus such as a metronome or a song to supplement rhythmic cueing (Thaut et al., 1996; Dalla Bella et al., 2017). PD patients after training with RAS showed improvements in gait and stride length (Thaut et al., 1996; Dalla Bella et al., 2017), which in turn decrease rates of falling as a result of providing external, rhythmic support (Thaut et al., 2019).

Dance is an activity that relies heavily on music, specifically on entrainment to musical rhythm, and combines rhythmic auditory and visual cues to coordinate movement. Several case studies as well as quasi-randomized pilot trials have shown beneficial effects of dance on both motor and non-motor PD symptoms including balance, gait, quality of life, mood, and other cognitive measures (Hackney \& Earhart, 2010a; Hashimoto et al., 2015; McNeely et al., 2015; Lewis et al., 2016). These findings have inspired the development of dance programs for PD (https://danceforparkinsons.org). Dance interventions, such as classes in Argentine Tango, jazz, or classical ballet, have specifically been shown to improve both gait and balance in individuals with PD (Hackney \& Earhart, 2010; Dos Santos Delabury et al., 2017, Sharp \& Hewitt, 2014; de Natale et al., 2017; Hulbert et al., 2017). Notably, while patients with PD exhibited improvements in balance following a dance intervention, these improvements were not observed in PD patients who had completed a rote exercise program (Hashimoto et al., 2015), suggesting a unique role for dance in assuaging parkinsonian symptoms. These findings are clinically important as problems with gait and balance are both very common in PD, and are also associated with increased rates of falls and other adverse events that decrease quality of life in older adults more generally 
(Creaby \& Cole, 2018). Because of their effectiveness, dance intervention has been posited to benefit even other older adults beyond those with PD (Patterson et al., 2018).

\subsection{Individual differences in neural structure and function impact rhythm perception and production}

The neural mechanisms supporting these benefits are likely related to the effects of music and dance on brain structure and function, and while the evidence supporting the beneficial impact of music- and dance-based interventions on PD is strong, responses to these interventions may vary based on individual differences in rhythm perception and production. Dalla Bella, et al.'s (2017) report that RAS improved gait in PD patients also found that the degree of improvement was dependent on patients' performance during a simple tapping task. Thus, the ability to entrain movements to music - sensorimotor coupling ability — was a predictor of therapeutic benefit from RAS. Furthermore, this finding suggests that objective rhythm tests, such as a simple tapping test to assess sensorimotor coupling ability, may be associated with improvements in PD symptoms.

Sensorimotor experience and ability in healthy adults also affect individual differences in neural structure and function. Having received formal training in music, for example, has been found to impact grey matter structure in the premotor and supplementary motor areas (Schneider et al., 2005; Gaser \& Schlaug, 2003; Grahn \& Rowe, 2009; Bermudez et al., 2009; Chaddock-Heyman et al., 2020). People with musical training also show superior beat perception compared with those without musical training, with concomitant underlying differences in functional connectivity between premotor and striatal areas (Grahn \& Rowe, 2009).

Response to music is also impacted by subjective experience: while the tendency to move one's body when music is playing is often unconscious, the degree of spontaneous movement to a certain piece of music may depend on a combination of musical or acoustic factors (syncopation, tempo) and subjective measures (enjoyment, familiarity). Distinct responses to different aspects of rhythm contribute to the perception of temporal structure in music. As these patterns typically obey a regular structure, the progression of a given rhythm is predictable (Schaefer et al., 2011). Interestingly, there is evidence to support the notion that the ability to predict a sequence of beats or the rhythmic progression of a melody is related to the extent to which a listener enjoys a certain piece of music (Schaefer et al., 2011). In this sense, the perception of rhythm, meter, and beat are important not only for music processing, but also for emotional and psychological responses to music. This is supported by work on musical groove, the pleasurable drive towards movement when listening to music, which is shown to impact sensorimotor experience (Janata et al., 2012; Witek et al., 2014). When asked to tap along to different songs, healthy participants show the most spontaneous motor excitability to high-groove songs, as compared with songs categorized as low-groove (Stupacher et al., 2013). Applied to PD, this suggests that groove may be a factor that can influence responsiveness to dance interventions, due to its effect on spontaneous motor excitability.

Dance experience has also been related to differences in patterns of neural activation, and is found to engage brain areas implicated in movement, especially rhythmic movement. When dancers observe others dancing, they show greater activity in the frontoparietal action observation network (AON), a circuit involved in the observation and production of movement (Calvo-Merino et al., 2005). The degree of 
AON activation is related to the familiarity of the task being observed, suggesting experience-dependent specificity (Calvo-Merino et al., 2005). Dancers have also shown increased functional connectivity in cortico-striatal pathways that are implicated in posture, movement, and action selection (Burzynska et al., 2017), enhanced white matter diffusivity (Karpati et al., 2018), and enhanced neuroplasticity in motor regions (Hanggi et al., 2010). These findings suggest that dance experience impacts the structure and function of brain regions involved in both the observation and production of movement.

Training in both music and dance, then, have measurable effects on the structure and function of brain regions involved in rhythm perception and production. However, it is still unclear how these experiences, in addition to variations in musical groove, might impact the effect of dance-based interventions on PD patients.

\subsection{Current Study and Hypothesis}

The current study aims to assess and predict the effects of four months of dance classes on parkinsonian symptoms. Specifically, we examine factors that could influence individual differences in responsiveness to dance classes. While studies have demonstrated that dance classes for PD benefit some disease symptoms, they have not assessed improvement in PD symptoms in conjunction with objective tests of rhythmic behavior. Rhythmic tapping behavior was previously found to be associated with the therapeutic benefit of auditory cueing in a PD population (Dalla Bella et al, 2017). Here, we ask whether these associations are preserved in the setting of dance classes. We investigated the effects of four months of weekly dance classes for PD on disease severity using the Unified Parkinson's Disease Rating Scale (UPDRS). We also assessed sensorimotor experience, musical groove, and prior experience with music and dance in order to determine whether these factors influence the therapeutic outcome of dance classes. In order to assess objective rhythm capabilities, we expanded the tapping task to include varying levels of groove, and added the Beat Alignment Test (BAT) to test beat perception and identification (Iversen \& Patel, 2008). With this study design, we tested the hypothesis that first, dance classes for PD improve disease symptoms and second, that groove and sensorimotor experience, as quantified by participants' prior experience with music and dance, affect the therapeutic outcome of these classes.

\section{Methods}

\subsection{Participants}

\section{Recruitment}

Participants, ranging in age from 59-84 $(n=30)$, were recruited from PD dance classes in New York City, NY $(n=18)$, San Rafael, CA $(n=7)$, and Santa Rosa, CA $(n=$ 5), and included 7 men and 23 women. Members of the control group were recruited from acquaintances and spouses of PD participants and included 8 men and 11 women. Control participants $(n=19)$ were matched for age, handedness, music experience $(M E)$, and dance experience (DE). Participants were considered to have ME or DE if they had over one year of formal training in some form of music or dance, respectively. All 
participants provided informed consent as approved by the Institutional Review Board of Wesleyan University.

\section{General Design (Pre- \& Post-Intervention Sessions)}

All 30 PD participants and 19 control participants completed the pre-intervention interview. This interview included four tasks: a questionnaire, the UPDRS (PD participants only), the BAT, and a tapping task. 20 PD participants completed the postintervention interview, which included the UPDRS, the BAT, and the tapping task. Postintervention data could not be collected in three participants because of death $(n=2)$ and a broken hip $(n=1)$. Five participants were unable to be contacted post-intervention, resulting in further loss to follow-up. Finally, two participants failed exclusion criteria for the post-intervention interview, so their data was not included in post-intervention analyses. There was no significant difference between baseline UPDRS scores for PD participants who did and did not complete the post-intervention interview $(\mathrm{t}(11.5)=1.30$, $p=0.218$ ), indicating that there was no self-selection for post-intervention testing.

\section{Inclusion / Exclusion criteria}

Inclusion criteria for members of the experimental group included a Parkinson's diagnosis, consistent weekly attendance of PD dance classes, and the ability to perform the tasks included in the study. Participants either had to be free of hearing impairments or, if they did have impairment, use a hearing aid during the study. Participants also had to be able to tap with one finger to complete the tapping task. Exclusion criteria for the post-intervention interview included the initiation of a new pharmaceutical or surgical intervention during the four-month course of the study, and the discontinuation of dance classes between the pre- and post-intervention interviews. One participant failed the first exclusion criterion as a result of implementing new deep brain stimulation (DBS) settings and experiencing improved motor ability as a result. One participant failed the second criterion, having stopped taking dance classes following the baseline interview.

\subsection{Procedure}

\section{Neuropsychological Assessment Battery}

All participants completed a questionnaire on age, location, gender, handedness, hearing impairment, length of time on carbidopa/levodopa, other medications taken to treat PD, most recent dose of carbidopa/levodopa, music experience, and dance experience.

\section{UPDRS}

The UPDRS was completed by all PD participants and measured disease severity through assessments of both motor and non-motor symptoms. Total scores for the UPDRS range from 0-199, with a score of 0 indicating no disability and a score of 199 indicating the most severe disability. Each question is scored from 0-4. The UPDRS is divided into four sections: Section I measures Mentation, Behavior, and Mood and is scored out of a possible 16 points; Section II measures Activities of Daily Life and is scored out of 52 points; Section III, the Motor Examination, is scored out of 108 points; and Section IV measures Complications of Therapy and is scored out of 23 points.

PD participants completed the UPDRS during both the pre- and post-intervention interviews. For participants on carbidopa/levodopa who experienced distinct "on" (more 
active) and "off" (less active) phases due to medication cycles, they were always in the "on" phases during the assessments. No video recordings of the UPDRS were taken because participants did not consent to being videotaped.

\section{BAT}

The BAT tests beat perception, capturing individual differences in beat processing. For the current study, twelve music samples were selected from the Iversen and Patel paper (2008) and played for all participants. A beep track was overlaid on the music in each track after five seconds. The onset of the beep track was timed so that it either directly coincided with the underlying beat (same phase and same frequency), or was $10 \%$ faster or slower than the rhythm of the music. Of the twelve music samples, five were "on the beat" and seven were "off the beat" (SM Table 1). Participants were asked to identify whether the beep track was on or off the beat and to rate their confidence in each answer.

\section{Tapping Task}

Participants completed a simple tapping task to assess sensorimotor coupling ability. Eight songs were selected from Janata, Tomic, and Haberman's groove index (Janata et al., 2012), which quantified musical groove on a scale of 0-127: "Cheek to Cheek", "In the Mood", "Sing Sing Sing", "Superstition", "Carolina in my Mind”, "Comfortably Numb", "'Til There was You", and "What a Wonderful World." The first four songs listed were designated as "high-groove" (Mean groove index $=97.4, \mathrm{SD}=9.4$ ) and the last four as "low-groove" (Mean groove index $=52.0, \mathrm{SD}=10.2)$. All "high-groove" songs were similarly designated as high-groove per Janata, et al., 2012, and all the "lowgroove" songs were originally designated as either mid or low-groove (SM Table 2). Each song was imported into GarageBand version 10.3.2 on a MacBook Pro and edited to a 30 second excerpt.

A KORG nanoPAD2 used to record participants' tapping. Participants were instructed to use one finger on their dominant hand to tap along to the beat of the music sample. They were asked to begin tapping as soon as they identified a beat of their choosing, and to continue tapping until the music stopped. The tapping track was then isolated and converted to a Wav file for analysis. After tapping to each excerpt, participants were asked to rate their enjoyment of and familiarity with the song on a scale of 1 (least enjoyable, least familiar) to 3 (most enjoyable, most familiar).

\subsection{Data Analysis}

\section{UPDRS}

Data were analyzed in RStudio (version 1.1.456) and MATLAB (The MathWorks, Inc., Natick, MA, version R2018a). Post-intervention UPDRS scores $(n=20)$ were subtracted from pre-intervention UPDRS scores to determine any changes in disease severity. Z-scores were calculated for each change in UPDRS score to normalize skewed distributions and analyzed using one-way ANOVA and Spearman's correlation. Missing data due to loss to follow-up $(n=10)$ resulted in fewer degrees of freedom for statistical analyses assessing changes in UPDRS scores than in analyses relying on data from preintervention interviews. 


\section{BAT}

False alarm rates (FAR) and hit rates (HR) were calculated from participants' categorizations of musical samples as "on" or "off" the beat. Average FAR (PD $=0.242$, control $=0.150)$ and HR $(P D=0.773$, control $=0.843)$ were calculated for both experimental and control groups (Results Table 1). Z-scores for HR and FAR were then calculated and participants' FAR z-scores were subtracted from their HR z-scores to get d', a measure of sensitivity. D' was used as a measure of BAT performance, as higher d' values indicated greater sensitivity to changes in stimuli.

\section{Tapping Task}

Audio files of recorded tapping were loaded into Matlab, where the MIDI Toolbox (Lartillot \& Toiviainen, 2007) was used to produce a waveform representation of each tapping track. Every waveform was then analyzed to determine the onset of tapping events. Inter-onset intervals (IOI) were computed from tapping events. The first three IOI values for each tapping recording were omitted from analysis to allow a period of time during which participants were becoming accustomed to the task. IOI values $>3$ seconds were also omitted from analysis (affecting 59 out of 552 total trials), as values this high were reflective of either a misunderstanding of the task or a failure of the KORG nanoPAD2 to register a tapping event (this affected $12.1 \%$ of pre-intervention PD trials, $12.5 \%$ of post-intervention PD trials, and $6.6 \%$ of control trials). Entropy, a measure of the randomness of events, was calculated for each trial of the tapping task. Entropy was computed by first extracting a polar histogram, set to 400 bins, from tapping IOIs for each trial to identify the frequency of events in each distinct bin. Then, Shannon entropy (Shannon, 1948) was computed for the histograms. Entropy was then analyzed using linear mixed-effects models, enabling analyses of both random effects of participant ID and fixed effects of groove (high vs. low), time-point (pre- vs. post-intervention), group (PD vs. control), music experience, and dance experience. Significance levels for all analyses were denoted as following in the following text and in all figures: $*=p<0.05$, $* *=\mathrm{p}<0.01, * * *=\mathrm{p}<0.001$. 


\section{Results}

\begin{tabular}{|c|c|c|c|c|}
\hline \multicolumn{2}{|l|}{ Group } & \multicolumn{2}{|c|}{ PD } & Control \\
\hline \multicolumn{2}{|l|}{$\mathrm{N}$} & \multicolumn{2}{|c|}{30} & 19 \\
\hline \multicolumn{2}{|c|}{ N with Dance Experience (DE) } & \multicolumn{2}{|c|}{$\begin{array}{c}20(66.7 \%) \\
7.4 \pm 7.60\end{array}$} & $\begin{array}{l}12(63.1 \%) \\
15.1 \pm 15.1\end{array}$ \\
\hline \multicolumn{2}{|c|}{$\mathrm{N}$ with Musical Experience (ME) } & \multicolumn{2}{|c|}{$\begin{array}{c}19(63.3 \%) \\
23.4 \pm 22.9\end{array}$} & $\begin{array}{c}11(57.9 \%) \\
20 \pm 22.2\end{array}$ \\
\hline $\begin{array}{l}\mathrm{BAT} \\
(\mathrm{M} \pm \mathrm{SD})\end{array}$ & $\begin{array}{l}\text { Hit Rate } \\
\text { FA Rate } \\
\text { d' }\end{array}$ & \multicolumn{2}{|c|}{$\begin{array}{c}0.773 \pm 0.216 \\
0.243 \pm 0.156 \\
1.70 \pm 0.962\end{array}$} & $\begin{array}{c}0.843 \pm 0.147 \\
0.150 \pm 0.111 \\
2.29 \pm 0.795\end{array}$ \\
\hline $\begin{array}{l}\text { Tapping Task } \\
\text { CV }(\mathrm{M} \pm \mathrm{SD})\end{array}$ & $\begin{array}{l}\text { Overall } \\
\text { High Groove } \\
\text { Low Groove }\end{array}$ & $\begin{array}{c}\text { Initial } \\
0.203 \pm 0.123 \\
0.206 \pm 0.122 \\
0.200 \pm 0.133 \\
\end{array}$ & $\begin{array}{c}\text { Follow-up } \\
0.168 \pm 0.112 \\
0.166 \pm 0.116 \\
0.171 \pm 0.132 \\
\end{array}$ & $\begin{array}{l}0.148 \pm 0.095 \\
0.155 \pm 0.118 \\
0.142 \pm 0.092 \\
\end{array}$ \\
\hline UPDRS & $\begin{array}{l}\text { Section I: Mentation, Behavior and Mood } \\
\text { Section II: Activities of Daily Life } \\
\text { Section III: Motor Examination } \\
\text { Section IV: Complications of Therapy } \\
\text { Total }\end{array}$ & $\begin{array}{l}2.80 \pm 1.86 \\
12.2 \pm 7.31 \\
10.6 \pm 5.82 \\
3.80 \pm 3.84 \\
29.9 \pm 14.7\end{array}$ & $\begin{array}{l}2.00 \pm 1.18 \\
9.81 \pm 5.90 \\
6.00 \pm 4.00 \\
3.05 \pm 3.09 \\
20.9 \pm 11.6\end{array}$ & $\begin{array}{l}\text { N/A } \\
\text { N/A } \\
\text { N/A } \\
\text { N/A } \\
\text { N/A }\end{array}$ \\
\hline
\end{tabular}

Table 1. Summary of key results from the questionnaire, BAT, tapping task (pre and post intervention), and UPDRS (pre and post intervention).

\subsection{Demographics and neuropsychological assessments}

Participants in the PD and control groups were matched for age, handedness, music experience, and dance experience (Table 1). The majority of participants had either previous music or dance experience (PD: $n=25$; Control: $n=15$ ), while a minority had both (PD: $\mathrm{n}=14$; Control: $\mathrm{n}=8$ ).

Pre-intervention UPDRS scores ranged from 6 to $74($ Mean $=29.9, \mathrm{SD}=14.7)$, representing the variability of disease severity and symptoms in our sample (Table 1). Based on these results, participants generally struggled most with activities of daily life, which include speech, salivation, swallowing, writing, cutting food, dressing, hygiene, and walking. To assess how UPDRS scores changed over time, we calculated the difference between the pre- and post-intervention UPDRS scores of the 20 participants who had completed assessments at both time-points. All but two of these participants exhibited either no change in score post-intervention, resulting in a score difference of zero, or an improvement (decrease) in score, resulting in a negative difference. A paired t-test comparing pre- and post-intervention UPDRS scores revealed significant differences between time-points $(\mathrm{t}(20)=4.81, \mathrm{p}=1.06 \mathrm{e}-04 * * *, \mathrm{~d}=0.507)$ (Figure 1). While the greatest number of participants $(n=17)$ saw an improvement in scores for UPDRS Section III, the Motor Assessment, there was general improvement across all sections of the UPDRS, with no significant differences between improvements across different sections of the UPDRS (SM Figure 1: one-way ANOVA and Tukey's HSD post-hoc testing revealed adjusted $\mathrm{p}$ values all $>0.05)$. 


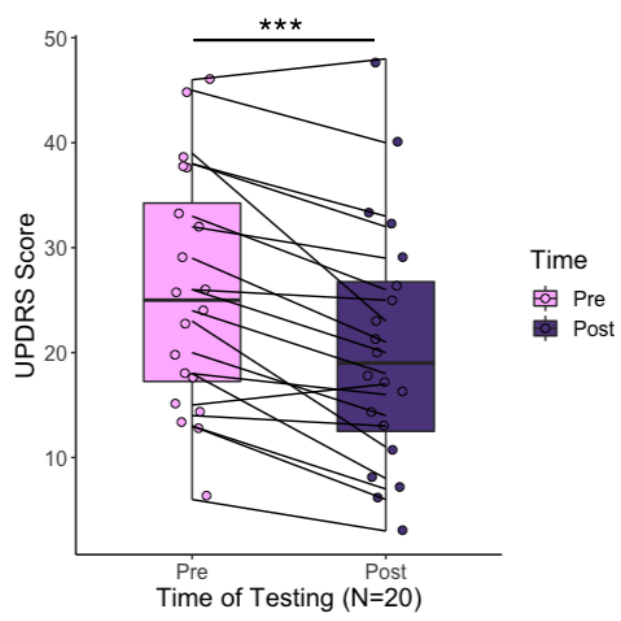

Figure 1. UPDRS scores pre-and post-intervention for the 20 PD participants who completed both interviews.

\subsection{Prior music experience benefits beat perception in PD}
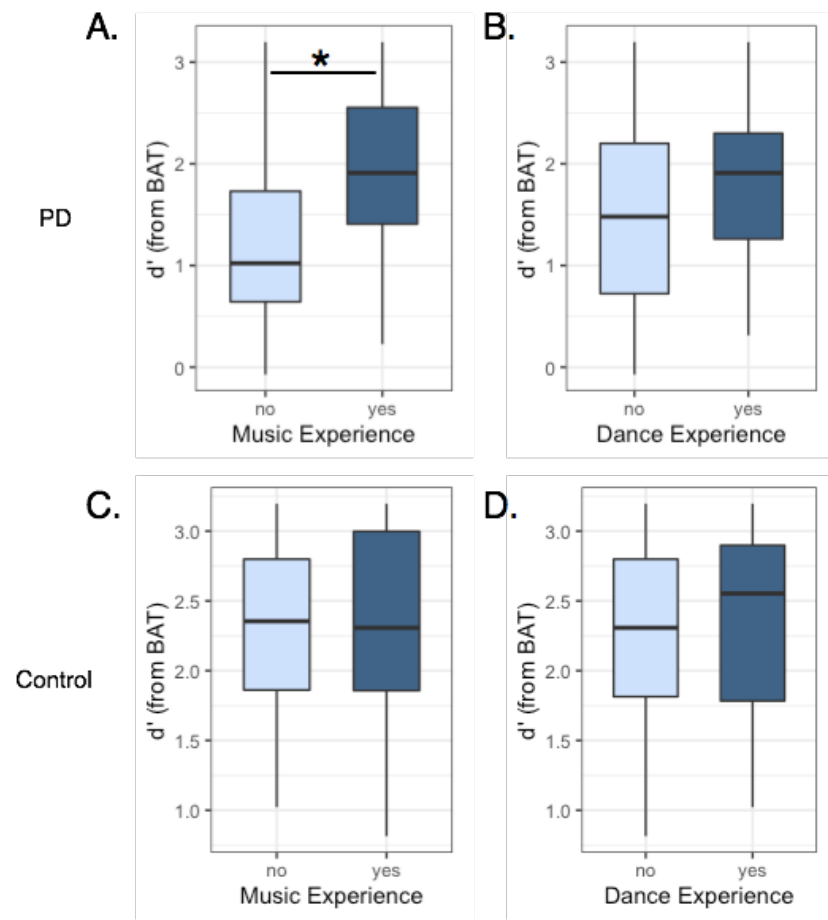

Figure 2. BAT results for $P D$ participants separated by $M E(A)$ and $D E(B)$, and for the control group, again separated by $M E(C)$ and $D E(D)$.

Results from the BAT revealed that control participants had higher average d' than PD participants (Control: 2.29; PD: 1.70, $\mathrm{t}(43.6)=-2.34, \mathrm{p}=0.0239 *, \mathrm{~d}=0.657$ ), demonstrating increased sensitivity to changes in beat and supporting previous work suggesting that PD impairs beat perception (Grahn, 2009).

D' measures of sensitivity were compared for PD and control groups by ME and DE to assess the effect of these experiences on beat perception (Figure 2). PD participants with ME performed significantly better on the BAT than PD participants without ME 
$\left[\mathrm{F}(1,28)=5.37, \mathrm{p}=0.0281^{*}, \mathrm{~d}=0.878\right.$, Figure $\left.2 \mathrm{~A}\right]$, while the presence of DE did not significantly affect BAT performance in PD participants $[F(1,28)=0.647, p=0.428, d=$ 0.312 , Figure 2B].

No significant differences were found between control participants with and without ME [One-way ANOVA: $F(1,17)=0.009, p=0.962, d=0.0438$, Figure $2 \mathrm{C}$ ] or between those with and without $\mathrm{DE}[\mathrm{F}(1,17)=0.080, \mathrm{p}=0.781, \mathrm{~d}=0.134$, Figure $2 \mathrm{D}]$.

\subsection{Effects of PD, groove, and time-point on tapping}

Figure 3 shows the distribution of intervals between tapping onsets, or inter-onset intervals (IOI), for the tapping task. Linear mixed-effects models (LMEs) were constructed to investigate whether Groove (High vs. Low), Group (PD vs. Control), and their interaction affected IOI values. First, a base model was constructed using IOI as the dependent variable and participant ID as a random effect. Then, Groove and Group were added as fixed effects. Model comparisons via likelihood ratio tests revealed that including Groove significantly improved model fit, $\chi^{2}(1)=5150, p<2.2 \mathrm{e}-16^{* * *}$, while including Group did not, $\chi^{2}(1)=1.18, p=0.279$. F-tests using Satterthwaite's method confirmed the significant main effect of Groove $\left[\mathrm{F}(1,16640)=6037, \mathrm{p}<2.2 \mathrm{e}-16^{* * *}\right]$, indicating higher IOIs for low-groove songs. The main effect of Group was not significant $[\mathrm{F}(1,46.5)=1.14, \mathrm{p}=0.291]$, but there was a significant interaction between Group and Groove $\left[\mathrm{F}(1,16640)=20.2, \mathrm{p}=7.07 \mathrm{e}-06^{* * *}\right]$, indicating that IOI values were higher in the low-groove condition for pre-intervention PD participants only. 


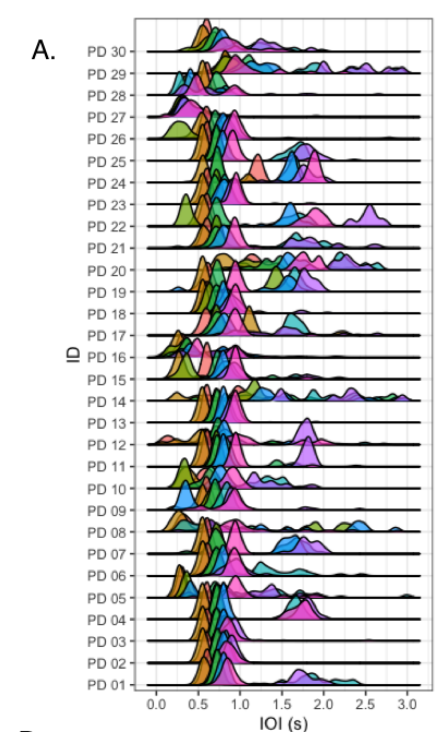

D.

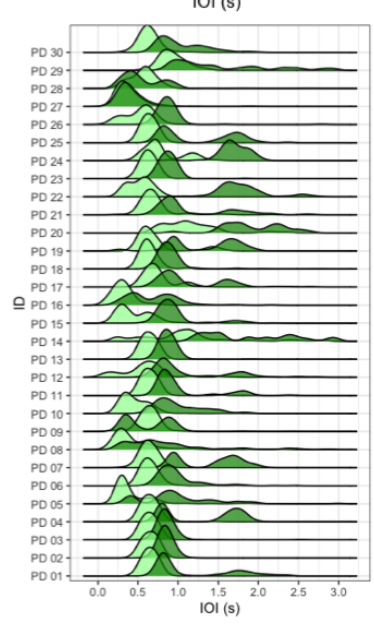

B.

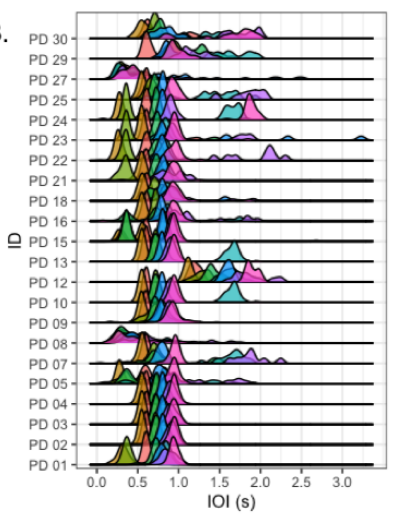

E.

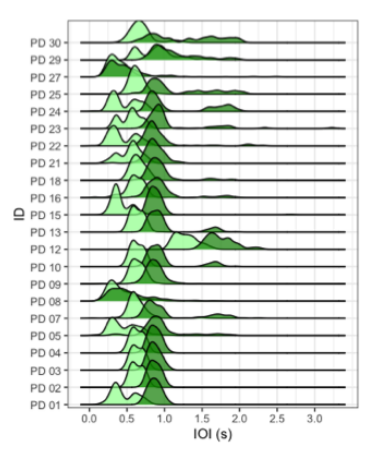

c.

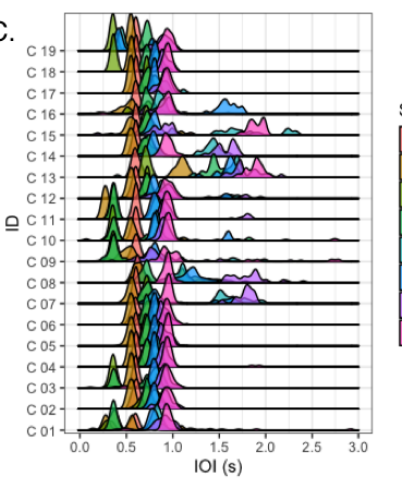

SONG

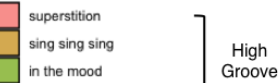

cheek to cheek

what a wonderful world

carolina in $\mathrm{my}$ mind

til there was you

comfortably numb
F.

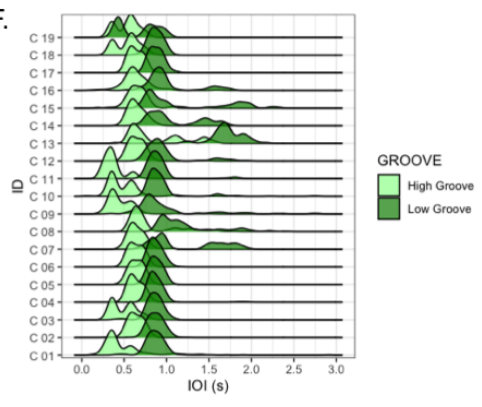

Figure 3. A. Distribution of pre-intervention PD inter-onset intervals (IOI) for tapping to each song. B. Distribution of post-intervention PD IOI values. $\boldsymbol{C}$. Distribution of control IOI values. D. Pre-intervention PD IOI distributions by groove. E. Post-intervention PD IOI distributions by groove. $\boldsymbol{F}$. Control IOI distributions by groove.

We next compared log(Entropy) values of PD participants' tapping pre- and postintervention (Figure 4A). Greater entropy values reflected increased randomness in tapping events, which we interpreted as a decrease in beat tracking accuracy and sensorimotor coupling ability. A base LME model was constructed with $\log$ (Entropy) as the dependent variable and participant ID as a random effect. Then, Time-Point (pre- vs. post-intervention) and Groove (high vs. low) were added as fixed effects. Likelihood ratio tests comparing the base model with models including these fixed effects revealed that including both Time-Point, $\chi^{2}(1)=9.75, p=0.00179 * *$, and Groove, $\chi^{2}(1)=10.6, p$ $=0.00113^{* *}$, independently increased model fit. F-tests using Satterthwaite's method confirmed the main effects of Time-Point $\left[\mathrm{F}(1,392)=9.85, \mathrm{p}=0.00183^{* *}\right]$ and Groove $\left[\mathrm{F}(1,378)=10.7, \mathrm{p}=0.00116^{* *}\right]$ indicating that post-intervention PD $\log ($ Entropy) values were lower than pre-intervention values, and that high-groove entropy values were 
lower than low-groove entropy (Figure 4B). F-tests revealed no significant interactions between Time-Point and Groove $[\mathrm{F}(1,374)=0.0472, \mathrm{p}=0.828]$.

To evaluate the potential differences between PD and controls in entropy separately for each time-point, we compared the base LME model (again with $\log$ (Entropy) as dependent variable and participant ID as random effect) against one with the fixed effect of Group (PD vs. control). Separate models were constructed using 1) pre-intervention PD and control entropy values, and 2) post-intervention PD and control entropy values.

For models comparing pre-intervention PD and control $\log$ (Entropy) values, likelihood ratio tests revealed that adding Group, $\chi^{2}(1)=7.51, p=0.00612 * *$ and Groove, $\chi^{2}(1)=9.48, p=0.00208 * *$ significantly and independently improved model fit. F-tests confirmed the main effects of Group $\left[\mathrm{F}(1,47)=7.79, \mathrm{p}=0.00757^{* *}\right]$ and Groove $\left[\mathrm{F}(1,342)=9.58, \mathrm{p}=0.00213^{* *}\right]$, indicating that pre-intervention PD $\log$ (Entropy) values were significantly higher than control values, and again that high-groove entropy values were lower than low-groove values. There were no significant interactions between Group and Groove $[\mathrm{F}(1,341)=0.200, \mathrm{p}=0.655]$.

The same process for models comparing post-intervention PD and control $\log (\mathrm{CV})$ values revealed that including only Groove, $\chi^{2}(1)=12.6, p=0.000393 * * *$, and not Group, $\chi^{2}(1)=1.88, p=0.170$, significantly improved model fit. F-tests revealed a significant main effect of Groove $[\mathrm{F}(1,279)=12.8, \mathrm{p}=0.000408 * * *]$, indicating again that high-groove entropy values were lower than low groove values. All other fixed effects and interaction terms were non-significant (all $p$ values $>0.2$ ). Together, these results demonstrate that PD participants reduced their tapping variability after the intervention, with tapping entropy more similar to that of controls. Furthermore, while tapping entropy varied across songs, high-groove entropy was consistently lower than low-groove entropy. 
A.

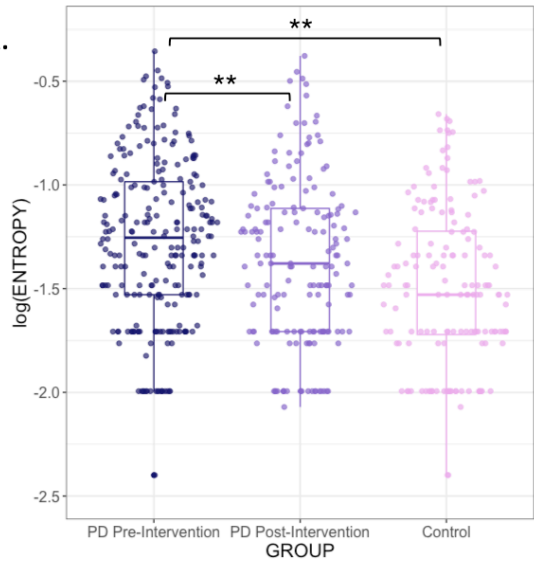

C.

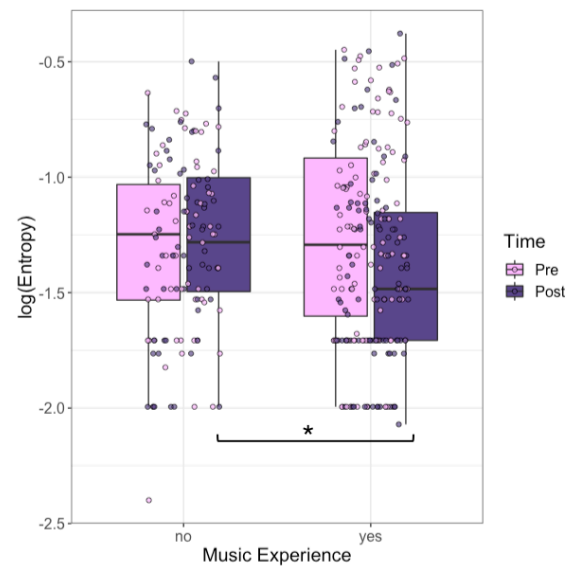

B.

GROUP

审 PD Post-Intervention

Control

Pre

Post

Figure 4. A. Comparison of log(Entropy) values for pre-intervention PD participants, post-intervention PD participants, and control participants. B. Figure $4 A$ by groove. $\boldsymbol{C}$. Log(Entropy) values by time of testing and music experience. D. Pre- and postintervention log(Entropy) values separated by groove, time of testing, and dance experience.

We then analyzed $\log ($ Entropy) values for PD participants by Groove, Time-Point, ME, and DE (Figure 4C,D). The base LME model for these analyses included participant ID as a random effect and $\log$ (Entropy) as the dependent variable. Groove, Time-Point, $\mathrm{ME}$, and DE were added as fixed effects. Adding Time-Point, $\chi^{2}(1)=9.75, \mathrm{p}=$ $0.00179^{* *}$, Groove, $\chi^{2}(1)=10.6, p=0.00113^{* *}$, and DE, $\chi^{2}(1)=9.02, p=0.00267^{* *}$, to the base model improved model fit; adding ME, $\chi^{2}(1)=0.339, p=0.561$, had no effect on model fit (SM Figure 2). F-tests revealed the significant main effect of DE $[F(1,27)=$ $\left.9.33, \mathrm{p}=0.00501^{* *}\right]$, indicating that those with $\mathrm{DE}$ exhibited lower tapping entropy than those without, and secondary effects of Time-Point $\left[\mathrm{F}(1,384)=5.52, \mathrm{p}=0.0193^{*}\right]$ and Groove $\left[\mathrm{F}(1,366)=5.36, \mathrm{p}=0.0211^{*}\right]$, indicating that entropy for both groove levels decreased post-intervention, but that low-groove entropy was consistently higher than high-groove entropy. F-tests also revealed significant interactions between Time-Point and $\operatorname{ME}\left[\mathrm{F}(1,384)=7.44, \mathrm{p}=0.00667^{* *}\right]$, where post-intervention entropy values were 
lower than pre-intervention values for participants with $\mathrm{ME}$, but not for those without it $\left[\mathrm{t}(334)=-2.30, \mathrm{p}=0.0219^{*}, \mathrm{~d}=-0.251\right]$ (Figure 4C), and between DE and Groove $\left[\mathrm{F}(1,366)=4.77, \mathrm{p}=0.0296^{*}\right]$ (Figure 4D).

Post-hoc analysis using Tukey's HSD test revealed significant differences between pre-intervention $\log$ (Entropy) values for PD participants with and without DE for both high-groove songs (diff $=-0.464, \mathrm{lwr}=-0.716$, upr $=-0.212, \mathrm{p}$ adj $=1.10 \mathrm{e}-06^{* * *}, \mathrm{~d}=$ 1.21 ) and low-groove songs (diff $=-0.352$, lwr $=-0.604$, upr $=-0.100, \mathrm{p}$ adj $=6.84 \mathrm{e}-$ $04 * * *, d=1.05)$. Significant differences were also found between post-intervention $\log$ (Entropy) values for PD participants with and without DE for high-groove songs (diff $=-0.332, \mathrm{lwr}=-0.584, \mathrm{upr}=-0.0805, \mathrm{p}$ adj $\left.=0.00179^{* *}, \mathrm{~d}=0.847\right)$, but not for lowgroove songs $($ diff $=-0.246, \mathrm{lwr}=-0.498, \mathrm{upr}=0.00558, \mathrm{p}$ adj $=0.0605, \mathrm{~d}=0.694)$ (Figure 4D).

\subsection{UPDRS improvement is associated with sensorimotor coupling ability}

We next assessed the relationship between $\log$ (Entropy) and improvements in UPDRS scores. We correlated the z-scores of participants' improvements in UPDRS scores with pre-intervention $\log$ (Entropy) values to assess the relationship between sensorimotor coupling ability and improvements in parkinsonian symptoms (Figure 5A). Baseline (pre-intervention) $\log$ (Entropy) was positively correlated with z-scores of UPDRS changes for both high-groove (Pearson's correlation: $r=0.302, p=0.00518^{* *}$ ) and low-groove $\left(\mathrm{r}=0.382, \mathrm{p}=0.000336^{* * *}\right)$ songs. Those who tapped with lower entropy during pre-intervention testing showed more improvement in PD symptoms. Similar patterns were found in correlations of post-intervention PD $\log$ (Entropy) values and z-scores of UPDRS changes (Figure 5B) (high-groove: $r=0.305, p=0.00498 * *$; low-groove: $\mathrm{r}=0.363, \mathrm{p}=0.000683 * * *)$. Thus, low tapping variability was a significant predictor of reduction in PD symptoms before dance intervention, but not after dance intervention.

We followed up on the relationship between UPDRS change and DE, as well as ME, using paired t-tests. PD participants with DE exhibited significantly greater improvements in UPDRS scores than those without $\left(\mathrm{t}(17.9)=2.80, \mathrm{p}=0.0119^{*}, \mathrm{~d}=\right.$ 1.16) (Figure 5E). This difference in UPDRS improvements was not observed when comparing participants with and without $\operatorname{ME}(\mathrm{t}(9.46)=-0.234, \mathrm{p}=0.820, \mathrm{~d}=0.121)$ (Figure 5D). 
A.

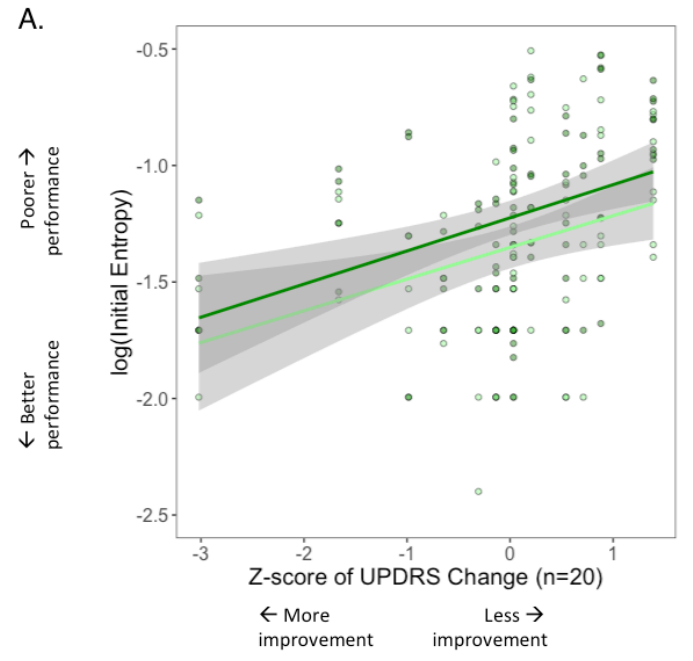

C.

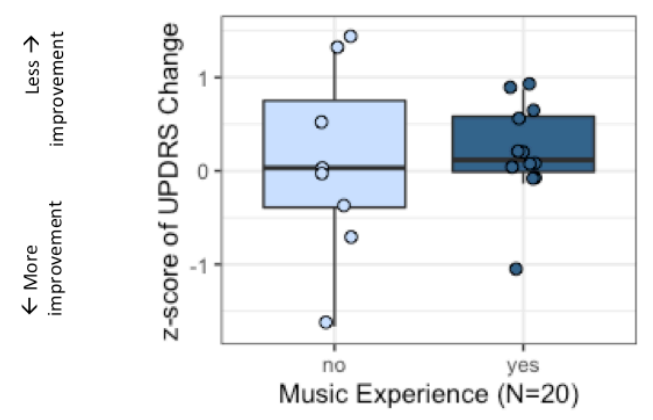

B.

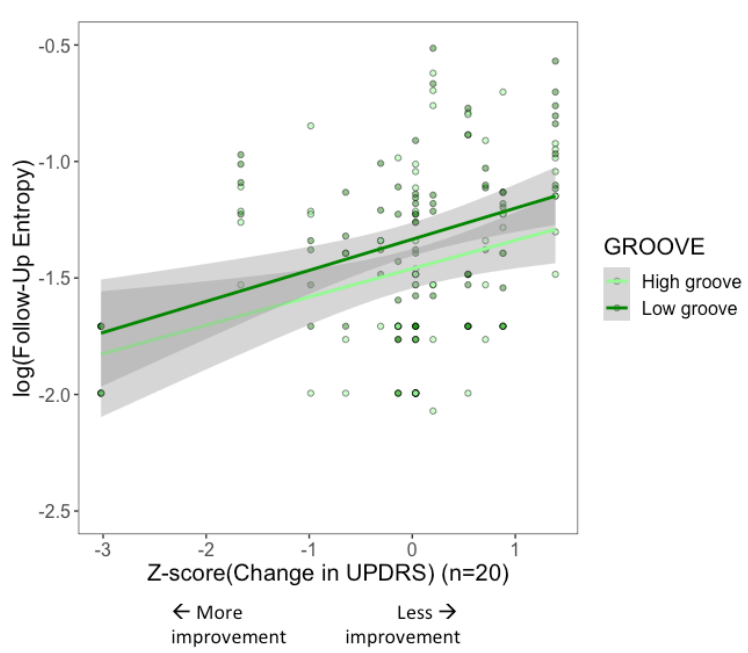

D.

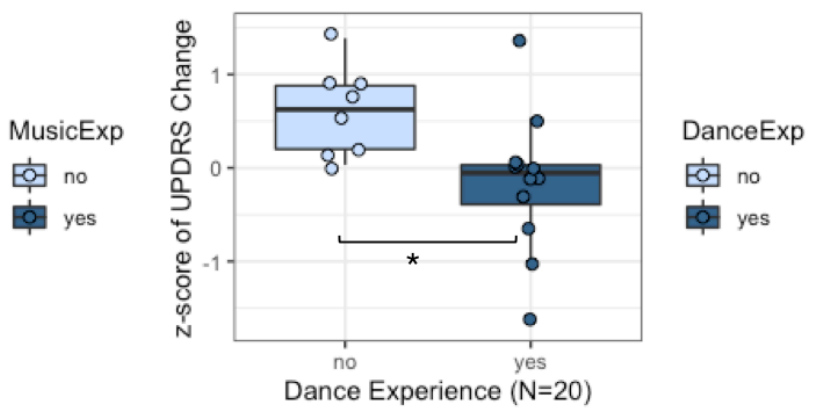

Figure 5. A. Relationships between entropy of pre-intervention tapping data and z-scores of changes in UPDRS from baseline to four months, for high-groove and low-groove trials. B. Relationships between entropy of post-intervention tapping data and z-scores of changes in UPDRS from baseline to four months, for high-groove and low-groove trials. C. Z-scores of changes in UPDRS from baseline to four months, separated by ME. D. Zscores of changes in UPDRS from baseline to four months, separated by DE.

\section{Discussion}

Results showed that dance classes were associated with reduced PD symptoms, and that dance experience and sensorimotor coupling ability (as assessed by rhythmic tapping) both contributed to the effectiveness of dance classes in reducing PD symptoms. While previous studies have demonstrated that dance classes for PD improve PD symptoms, they have focused primarily on the classes and symptoms themselves through investigating partnered versus non-partnered movement (Hackney \& Earhart, 2010b), comparisons between different dance styles (Hackney \&Earhart, 2009), assessments of gait and balance (Hackney \& Earhart, 2010a; Rios Romenets et al., 2015; Hashimoto et al., 2015; de Natale et al., 2017), mood and quality of life measures (Lewis et al., 2014; Hashimoto et al., 2015; McNeely et al., 2015), and the effects of dance intervention on 
specific aspects of movement coordination (Hulbert et al., 2017). To our knowledge, this is the first study to employ objective rhythm tests, and to assess previous training in music and dance as predictors of individual differences in responsiveness to PD dance classes. By comparing participants with and without musical and dance experience, and including measures of beat perception, response to musical groove, and tapping ability, the present study aims not only to assess the outcomes of dance classes on PD symptoms, but also to predict these outcomes using behavioral measures before and after dance intervention.

Our BAT results support previous findings that PD individuals exhibit impaired beat perception and sensitivity when compared with healthy controls (Grahn, 2009). This impairment is likely explained by impaired basal ganglia and motor activity and connectivity, which are involved in beat perception ability (Grahn \& Rowe, 2009). Contrary to previous reports (Grahn \& McAuley, 2009), we did not find an effect of prior music experience on sensitivity in beat perception among healthy controls; however, we did find an effect of musical experience on sensitivity in beat perception among PD participants. The different pattern of results between PD and controls may be due to the relatively small sample size of our control group; alternately, it could be due to a relative ceiling effect among controls, as both musically experienced and inexperienced control participants performed well above chance and better than PD participants on the BAT. From these results, it appears that the effects of musical experience only emerged when neural circuitry supporting beat perception is compromised in PD. Prior musical experience may have been neuroprotective in that it equipped the PD participants to better identify changes in beat and rhythm.

Tapping task results also showed experience-dependent patterns, although primarily dependent on dance and not music. As a group, PD participants improved significantly on the tapping task at post-intervention testing; however, this improvement was larger for those without dance experience. This was likely due to better performance among the participants with DE before intervention, as PD participants with DE performed strongly and consistently across time-points. Those without DE performed worse during preintervention testing, but improved post-intervention to the point where their high-groove tapping was more similar to those with DE, and there were no longer any significant DEdependent differences in post-intervention low-groove tapping. Decreased entropy leads to increased predictability of rhythm (Milne \& Herff, 2020), and because variability in tapping performance is associated with superior sensorimotor coupling ability (Merchant et al, 2011; Karpati et al, 2016), lower tapping entropy in PD participants with prior dance experience may again suggest a neuroprotective effect of dance training. PD participants with music experience similarly demonstrated an improvement in tapping entropy during post-intervention testing compared to pre-intervention testing; this may compared to pre-intervention testing; this may reflect utilization of the audiomotor circuitry underlying this group's enhanced beat perception ability.

DE-dependent performance on tapping was also found to be predictive of postintervention improvements in UPDRS. Correlations between pre-intervention tapping entropy and changes in UPDRS scores suggest that enhanced-less random, more predictable - sensorimotor coupling ability was associated with larger improvements in disease symptoms. This finding is similar to previous reports associating tapping task performance and therapeutic outcome following RAS in individuals with PD (Dalla Bella 
et al., 2017). Increased therapeutic benefit from Parkinson's dance classes in individuals with prior dance training may be reflective of changes in structure and function in brain regions involved in dancing, such as motor and premotor regions and the corticostriatal network (Brown et al., 2006), which is also disrupted in PD (Kalia and Lang, 2015). Dance training — and to perhaps a lesser extent, music training — may strengthen neural pathways involved in motor control, auditory-motor entrainment, action observation, and/or kinesthetic or proprioceptive feedback mechanisms that could then be utilized as an alternative to impaired motor areas. This training-dependent strengthening could in turn prime PD individuals with DE to be more responsive to dance intervention, while being more stable over time in tapping tasks. While participants with DE performed consistently in tapping tasks, participants without DE exhibited more improvement in tapping, which may suggest more plasticity in sensorimotor coupling ability. This pattern of results could reflect multiple mechanisms in which dance helps PD: both as a longterm neuroprotective agent of underlying motor and corticostriatal circuits, and as a shorter-term facilitator or primer of more stable motor behavior.

While DE generally improved tapping as shown in decreased entropy for all trial types among those with DE, ME showed an interaction with time-point in that participants with ME showed more improvement at the post-intervention testing. This may be because ME predisposes individuals to react better to the dance intervention, or it may suggest that those with ME adapt more quickly to the tapping task. The present results could not disentangle between an effect of intervention and an effect of general improvement over the course of repeated testing. Future studies should implement a control intervention to tease apart these competing interpretations.

Despite this complex relationship between pre-intervention tapping stability and therapeutic benefit from dance classes, the indication of learning in PD participants without $\mathrm{DE}$ and in participants with $\mathrm{ME}$ raises questions about how improvements in sensorimotor coupling may affect responses to dance classes over longer periods of time.

Another factor that may also play a role in explaining our findings is cognitive reserve, i.e. experiential factors that affect individual differences in participants' ability to resist or compensate for behavioral and/or cognitive declines in later life (Tucker-Drob et al., 2009), and have been recently associated with cognitive and motor function in PD (Lee et al, 2019). In this regard, dance experience may well serve as an experiential factor that builds cognitive reserve, as dance is a cognitively demanding experience that is also a relatively common leisure activity among older adults (Rodrigues-Krause et al, 2019). Although we did not test this directly, the therapeutic outcome of dance classes may also be associated with socialization and sense of community (Rocha et al, 2017). Incorporating movement, relaxation, and social interaction with music could further decrease stress and anxiety and, consequently, neuroinflammation and oxidative stress (Knight \& Rickard, 2001; Kuo et al., 2015; Bhasin et al., 2018; Jensen \& Bonde, 2018). These potential side benefits of dance merit further investigation as they may be used to better tailor dance therapy in the future.

While all PD participants in this study were tested around the same time of day (late morning and midday), in an "on" phase during testing, and on dopaminergic medication for PD, we did not control for individual differences in pharmacological routine. We also did not control for participation in additional therapies beyond the attendance of weekly dance classes because of the potential benefit other therapies may have provided. 
Because participants were uncomfortable with giving video consent during the study, we were unable to score the UPDRS using blinded videos, which may have introduced an observer bias to the UPDRS. Nevertheless, results from the tapping data and beat perception tests did not depend on observers or coding. However, there may have been test-retest effects, as participants could have been more comfortable with the tasks the second time around and therefore performed better during post-intervention testing.

Another caveat of these findings is the lack of random assignment and a control intervention: due to limitations in time and resources, we were only able to recruit participants who self-selected into dance intervention, and we were not able to compare dance intervention against a control intervention in this study. Although we had a wellmatched control group who were not affected by PD, this control group did not receive intervention, and was smaller in sample size than the PD group. Nevertheless, our healthy control group provided aged-matched control data for the BAT and the rhythmic tapping tasks, which showed better performance (superior beat perception and lower tapping variability) than the pre-intervention but not the post-intervention PD participants. Another limitation of the present results is that a third of the PD participants were lost to follow-up, for a variety of reasons as described in Methods. While concerns about selfselection were partly alleviated by finding similar performance between pre-intervention UPDRS scores for PD participants who did and did not complete the post-intervention tests, the issue of survivor bias remains, in that those who participated in postintervention testing could have been more engaged, better-supported, or otherwise better situated to improve in symptoms after intervention. While future studies are needed to eliminate the possibility of survivor bias, the current findings are not inconsistent with previous results in showing improvement in motor symptoms following dance intervention (Hackney \& Earhart, 2010a; Rios Romenets et al., 2015; Hashimoto et al., 2015; de Natale et al., 2017). While replicating these results, we also provide additional detailed data in support of rhythm and sensorimotor coupling as the underlying mechanisms that support improvements from dance intervention.

\section{Conclusions and Future Directions}

The present study tested the effects, and examined mechanistic predictors of success, of a dance intervention for PD. Our findings support the implementation of dance programs in PD communities, as dance classes create a supportive environment that can improve motor ability and decrease symptom severity. Our results also support involvement in dance for healthy individuals, as the presence of previous dance training significantly affected sensorimotor coupling, which in turn predicts the therapeutic outcome of movement therapy for PD.

Through the use of objective rhythm assessments, we found associations between previous dance experience, ability to predictably entrain movements to a musical beat, and improvements in PD symptoms following a dance intervention. Our study has implications for the mechanistic understanding of behavioral interventions for neurodegenerative disorders, and may inform individualized interventions to maximize therapeutic outcome of dance classes for PD. Looking ahead, it would be beneficial to extend the current study to invite more PD communities to participate. Adding a third follow-up session may also further elucidate the groove-related effects on sensorimotor 
experience, as well as the effects of dance classes on participants without previous dance training. Given the observed benefits of dance for PD in the present study, future studies should test dance interventions more widely to see if the observed benefits persist over time and across larger samples, and if these benefits may also apply to other disease populations.

\section{Author Contributions}

AK and PL: Conceptualization; AK: Data curation; Data analysis; PL: Funding acquisition; AK and PL: Investigation; Methodology; Project administration; Resources; Software; PL: Supervision; AK and PL: Visualization; Roles/Writing - AK: original draft; PL: Writing - review \& editing.

\section{Declaration of Funding}

Supported by Imagination Institute, Grammy Foundation, and NSF-CAREER \#1945436 to PL, and startup funds from Wesleyan University and Northeastern University.

\section{Acknowledgments}

Special thanks to Jessica Grahn for helpful advice on the design of this study, to Parker Tichko for help with statistical methods, to Shinya Fujii for helpful comments on data analysis, and to David Leventhal for his support of the project. 


\section{References}

Benoit, C. E., Dalla Bella, S., Farrugia, N., Obrig, H., Mainka, S., \& Kotz, S. A. (2014). Musically cued gait-training improves both perceptual and motor timing in Parkinson's disease. Front Hum Neurosci, 8, 494. doi:10.3389/fnhum.2014.00494

Bermudez, P., Lerch, J. P., Evans, A. C., \& Zatorre, R. J. (2009). Neuroanatomical correlates of musicianship as revealed by cortical thickness and voxel-based morphometry. Cereb Cortex, 19(7), 1583-1596. doi:10.1093/cercor/bhn196

Bhasin, M. K., Denninger, J. W., Huffman, J. C., Joseph, M. G., Niles, H., ChadFriedman, E., ... Libermann, T. A. (2018). Specific Transcriptome Changes Associated with Blood Pressure Reduction in Hypertensive Patients After Relaxation Response Training. J Altern Complement Med, 24(5), 486-504. doi:10.1089/acm.2017.0053

Brown, S., Martinez, M. J., \& Parsons, L. M. (2006). The neural basis of human dance. Cereb Cortex, 16(8), 1157-1167. doi:10.1093/cercor/bhj057

Burzynska, A. Z., Finc, K., Taylor, B. K., Knecht, A. M., \& Kramer, A. F. (2017). The Dancing Brain: Structural and Functional Signatures of Expert Dance Training. Front Hum Neurosci, 11, 566. doi:10.3389/fnhum.2017.00566

Calvo-Merino, B., Glaser, D. E., Grezes, J., Passingham, R. E., \& Haggard, P. (2005). Action observation and acquired motor skills: an FMRI study with expert dancers. Cereb Cortex, 15(8), 1243-1249. doi:10.1093/cercor/bhi007

Chaddock-Heyman, L., Loui, P., Weisshappel, R., McAuley, E., \& Kramer, A. F. (2020). Musical training and brain volume in older adults. In review.

Creaby, M. W., \& Cole, M. H. (2018). Gait characteristics and falls in Parkinson's disease: A systematic review and meta-analysis. Parkinsonism Relat Disord, 57, 1-8. doi:10.1016/j.parkreldis.2018.07.008

de Natale, E. R., Paulus, K. S., Aiello, E., Sanna, B., Manca, A., Sotgiu, G., . . Deriu, F. (2017). Dance therapy improves motor and cognitive functions in patients with Parkinson's disease. NeuroRehabilitation, 40(1), 141-144. doi:10.3233/NRE161399

Dalla Bella, S., Benoit, C. E., Farrugia, N., Keller, P. E., Obrig, H., Mainka, S., \& Kotz, S. A. (2017). Gait improvement via rhythmic stimulation in Parkinson's disease is linked to rhythmic skills. Sci Rep, 7, 42005. doi:10.1038/srep42005

Devlin, K., Alshaikh, J. T., \& Pantelyat, A. (2019). Music Therapy and Music-Based Interventions for Movement Disorders. Curr Neurol Neurosci Rep, $19(11), 83$. doi:10.1007/s11910-019-1005-0

Dos Santos Delabary, M., Komeroski, I. G., Monteiro, E. P., Costa, R. R., \& Haas, A. N. (2018). Effects of dance practice on functional mobility, motor symptoms and quality of life in people with Parkinson's disease: a systematic review with metaanalysis. Aging Clin Exp Res, 30(7), 727-735. doi:10.1007/s40520-017-0836-2

Gaser, C., \& Schlaug, G. (2003). Brain structures differ between musicians and nonmusicians. J Neurosci, 23(27), 9240-9245. 
Grahn, J. A. (2009). The role of the basal ganglia in beat perception: neuroimaging and neuropsychological investigations. Ann N Y Acad Sci, 1169, 35-45. doi:10.1111/j.1749-6632.2009.04553.x

Grahn, J. A., \& Rowe, J. B. (2013). Finding and feeling the musical beat: striatal dissociations between detection and prediction of regularity. Cereb Cortex, 23(4), 913-921. doi:10.1093/cercor/bhs083

Grahn, J. A., \& Brett, M. (2009). Impairment of beat-based rhythm discrimination in Parkinson's disease. Cortex, 45(1), 54-61. doi:10.1016/j.cortex.2008.01.005

Grahn, J. A., \& McAuley, J. D. (2009). Neural bases of individual differences in beat perception. Neuroimage, 47(4), 1894-1903.

doi:10.1016/j.neuroimage.2009.04.039

Hackney, M. E., \& Earhart, G. M. (2009). Effects of dance on movement control in Parkinson's disease: a comparison of Argentine tango and American ballroom. $J$ Rehabil Med, 41(6), 475-481. doi:10.2340/16501977-0362

Hackney, M. E., \& Earhart, G. M. (2010a). Effects of dance on balance and gait in severe Parkinson disease: a case study. Disabil Rehabil, 32(8), 679-684. doi:10.3109/09638280903247905

Hackney, M. E., \& Earhart, G. M. (2010). Effects of dance on gait and balance in Parkinson's disease: a comparison of partnered and nonpartnered dance movement. Neurorehabil Neural Repair, 24(4), 384-392. doi:10.1177/1545968309353329

Hanggi, J., Koeneke, S., Bezzola, L., \& Jancke, L. (2010). Structural neuroplasticity in the sensorimotor network of professional female ballet dancers. Hum Brain Mapp, 31(8), 1196-1206. doi:10.1002/hbm.20928

Hashimoto, H., Takabatake, S., Miyaguchi, H., Nakanishi, H., \& Naitou, Y. (2015). Effects of dance on motor functions, cognitive functions, and mental symptoms of Parkinson's disease: a quasi-randomized pilot trial. Complement Ther Med, 23(2), 210-219. doi:10.1016/j.ctim.2015.01.010

Hausdorff, J. M., Cudkowicz, M. E., Firtion, R., Wei, J. Y., \& Goldberger, A. L. (1998). Gait variability and basal ganglia disorders: stride-to-stride variations of gait cycle timing in Parkinson's disease and Huntington's disease. Mov Disord, 13(3), 428437. doi:10.1002/mds. 870130310

Hellstrom, A., \& Almkvist, O. (1997). Tone duration discrimination in demented, memory-impaired, and healthy elderly. Dement Geriatr Cogn Disord, 8(1), 49-54. doi:10.1159/000106600

Hulbert, S., Ashburn, A., Roberts, L., \& Verheyden, G. (2017). Dance for Parkinson'sThe effects on whole body co-ordination during turning around. Complement Ther Med, 32, 91-97. doi:10.1016/j.ctim.2017.03.012

Iversen, J. R., \& Patel, A. D. (2008). The Beat Alignment Test (BAT): Surveying beat processing abilities in the general population. International Conference on Music Perception and Cognition, 10, 465-468.

Jahanshahi, M., Jones, C. R., Dirnberger, G., \& Frith, C. D. (2006). The substantia nigra pars compacta and temporal processing. J Neurosci, 26(47), 12266-12273. doi:10.1523/JNEUROSCI.2540-06.2006 
Janata, P., Tomic, S. T., \& Haberman, J. M. (2012). Sensorimotor coupling in music and the psychology of the groove. J Exp Psychol Gen, 141(1), 54-75. doi: $10.1037 / \mathrm{a} 0024208$

Jensen, A., \& Bonde, L. O. (2018). The use of arts interventions for mental health and wellbeing in health settings. Perspect Public Health, 138(4), 209-214. doi:10.1177/1757913918772602

Karpati, F. J., Giacosa, C., Foster, N. E., Penhune, V. B., \& Hyde, K. L. (2016). Sensorimotor integration is enhanced in dancers and musicians. Exp Brain Res, 234(3), 893-903. doi:10.1007/s00221-015-4524-1

Knight, W. E., \& Rickard Ph, D. N. (2001). Relaxing music prevents stress-induced increases in subjective anxiety, systolic blood pressure, and heart rate in healthy males and females. J Music Ther, 38(4), 254-272.

Kuo, B., Bhasin, M., Jacquart, J., Scult, M. A., Slipp, L., Riklin, E. I., . . Denninger, J. W. (2015). Genomic and clinical effects associated with a relaxation response mind-body intervention in patients with irritable bowel syndrome and inflammatory bowel disease. PLoS One, 10(4), e0123861.

doi:10.1371/journal.pone.0123861

Lang, A. E., \& Lozano, A. M. (1998). Parkinson's disease. N Engl J Med, 339(15), 10441053. doi:10.1056/NEJM199810083391506

Lartillot, C., and Toiviainen, P. (2007). A Matlab toolbox for musical feature extraction from audio. Paper presented at the International Conference on Digital Audio Effects, Bordeaux, France.

Lee, P. C., Artaud, F., Cormier-Dequaire, F., Rascol, O., Durif, F., Derkinderen, P., .. . Group, D. S. (2019). Examining the Reserve Hypothesis in Parkinson's Disease: A Longitudinal Study. Mov Disord, 34(11), 1663-1671. doi:10.1002/mds.27854

Lewis, C., Annett, L. E., Davenport, S., Hall, A. A., \& Lovatt, P. (2016). Mood changes following social dance sessions in people with Parkinson's disease. J Health Psychol, 21(4), 483-492. doi:10.1177/1359105314529681

McNeely, M. E., Duncan, R. P., \& Earhart, G. M. (2015). Impacts of dance on non-motor symptoms, participation, and quality of life in Parkinson disease and healthy older adults. Maturitas, 82(4), 336-341. doi:10.1016/j.maturitas.2015.08.002

Meck, W. H. (1996). Neuropharmacology of timing and time perception. Brain Res Cogn Brain Res, 3(3-4), 227-242. doi:10.1016/0926-6410(96)00009-2

Meck, W. H., \& Benson, A. M. (2002). Dissecting the brain's internal clock: how frontalstriatal circuitry keeps time and shifts attention. Brain Cogn, 48(1), 195-211. doi:10.1006/brcg.2001.1313

Merchant, H., Zarco, W., Perez, O., Prado, L., \& Bartolo, R. (2011). Measuring time with different neural chronometers during a synchronization-continuation task. Proc Natl Acad Sci U S A, 108(49), 19784-19789. doi:10.1073/pnas.1112933108

Milne, A. J., \& Herff, S. A. (2020). The perceptual relevance of balance, evenness, and entropy in musical rhythms. Cognition, 203, 104233. doi:10.1016/j.cognition.2020.104233 
Mirelman, A., Bonato, P., Camicioli, R., Ellis, T. D., Giladi, N., Hamilton, J. L., . . Almeida, Q. J. (2019). Gait impairments in Parkinson's disease. Lancet Neurol, 18(7), 697-708. doi:10.1016/S1474-4422(19)30044-4

Nenadic, I., Gaser, C., Volz, H. P., Rammsayer, T., Hager, F., \& Sauer, H. (2003). Processing of temporal information and the basal ganglia: new evidence from fMRI. Exp Brain Res, 148(2), 238-246. doi:10.1007/s00221-002-1188-4

O'Boyle, D. J., Freeman, J. S., \& Cody, F. W. (1996). The accuracy and precision of timing of self-paced, repetitive movements in subjects with Parkinson's disease. Brain, 119 ( Pt 1), 51-70. doi:10.1093/brain/119.1.51

Pastor, M. A., Artieda, J., Jahanshahi, M., \& Obeso, J. A. (1992). Time estimation and reproduction is abnormal in Parkinson's disease. Brain, 115 Pt 1, 211-225. doi:10.1093/brain/115.1.211

Patterson, K. K., Wong, J. S., Prout, E. C., \& Brooks, D. (2018). Dance for the rehabilitation of balance and gait in adults with neurological conditions other than Parkinson's disease: A systematic review. Heliyon, 4(3), e00584. doi:10.1016/j.heliyon.2018.e00584

Poewe, W., Seppi, K., Tanner, C. M., Halliday, G. M., Brundin, P., Volkmann, J., . . Lang, A. E. (2017). Parkinson disease. Nat Rev Dis Primers, 3, 17013. doi: $10.1038 /$ nrdp. 2017.13

Rios Romenets, S., Anang, J., Fereshtehnejad, S. M., Pelletier, A., \& Postuma, R. (2015). Tango for treatment of motor and non-motor manifestations in Parkinson's disease: a randomized control study. Complement Ther Med, 23(2), 175-184. doi:10.1016/j.ctim.2015.01.015

Rocha, P. A., Slade, S. C., McClelland, J., \& Morris, M. E. (2017). Dance is more than therapy: Qualitative analysis on therapeutic dancing classes for Parkinson's. Complement Ther Med, 34, 1-9. doi:10.1016/j.ctim.2017.07.006

Rodrigues-Krause, J., Krause, M., \& Reischak-Oliveira, A. (2019). Dancing for Healthy Aging: Functional and Metabolic Perspectives. Altern Ther Health Med, 25(1), 44-63.

Rose, D., Cameron, D. J., Lovatt, P. J., Grahn, J. A., \& Annett, L. E. (2020). Comparison of Spontaneous Motor Tempo during Finger Tapping, Toe Tapping and Stepping on the Spot in People with and without Parkinson's Disease. J Mov Disord, 13(1), 47-56. doi:10.14802/jmd.19043

Schneider, P., Sluming, V., Roberts, N., Bleeck, S., \& Rupp, A. (2005). Structural, functional, and perceptual differences in Heschl's gyrus and musical instrument preference. Ann N Y Acad Sci, 1060, 387-394. doi:10.1196/annals. 1360.033

Sharp, K., \& Hewitt, J. (2014). Dance as an intervention for people with Parkinson's disease: a systematic review and meta-analysis. Neurosci Biobehav Rev, 47, 445456. doi:10.1016/j.neubiorev.2014.09.009 
Spencer, R. M., \& Ivry, R. B. (2005). Comparison of patients with Parkinson's disease or cerebellar lesions in the production of periodic movements involving event-based or emergent timing. Brain Cogn, 58(1), 84-93. doi:10.1016/j.bandc.2004.09.010

Stupacher, J., Hove, M. J., Novembre, G., Schutz-Bosbach, S., \& Keller, P. E. (2013). Musical groove modulates motor cortex excitability: a TMS investigation. Brain Cogn, 82(2), 127-136. doi:10.1016/j.bandc.2013.03.003

Thaut, M. H., McIntosh, G. C., Rice, R. R., Miller, R. A., Rathbun, J., \& Brault, J. M. (1996). Rhythmic auditory stimulation in gait training for Parkinson's disease patients. Mov Disord, 11(2), 193-200. doi:10.1002/mds.870110213

Thaut, M. H., Rice, R. R., Braun Janzen, T., Hurt-Thaut, C. P., \& McIntosh, G. C. (2019). Rhythmic auditory stimulation for reduction of falls in Parkinson's disease: a randomized controlled study. Clin Rehabil, 33(1), 34-43. doi: $10.1177 / 0269215518788615$

Tucker-Drob, E. M., Johnson, K. E., \& Jones, R. N. (2009). The cognitive reserve hypothesis: a longitudinal examination of age-associated declines in reasoning and processing speed. Dev Psychol, 45(2), 431-446. doi:10.1037/a0014012

Witek, M. A., Clarke, E. F., Wallentin, M., Kringelbach, M. L., \& Vuust, P. (2014). Syncopation, body-movement and pleasure in groove music. PLoS One, 9(4), e94446. doi:10.1371/journal.pone.0094446 